\title{
Does asymptotic simplicity allow for radiation near spatial infinity?
}

\author{
Juan Antonio Valiente Kroon * \\ Institut für Theoretische Physik, \\ Universität Wien, \\ Boltzmanngasse 5, A-1090 Wien, \\ Austria.
}

November 11, 2018

\begin{abstract}
A representation of spatial infinity based on the properties of conformal geodesics is used to obtain asymptotic expansions of the gravitational field near the region where null infinity touches spatial infinity. These expansions show that generic time symmetric initial data with an analytic conformal metric at infinity will give rise to developments with a certain type of logarithmic singularities at the points where null infinity and spatial infinity meet. These logarithmic singularities produce a non-smooth null infinity. The sources of the logarithmic singularities are traced back down to the initial data. It is shown that if the parts of the initial data responsible for the non-regular behaviour of the solutions are not present, then the initial data is static to a certain order. On the basis of these results it is conjectured that the only time symmetric data sets with developments having a smooth null infinity are those which are static in a neighbourhood of infinity. This conjecture generalises a previous conjecture regarding time symmetric, conformally flat data. The relation of these conjectures to Penrose's proposal for the description of the asymptotic gravitational field of isolated bodies is discussed.
\end{abstract}

PACS numbers: 04.20.Ha, 04.20.Ex.

\section{Introduction}

A central issue in the general relativistic theory of isolated systems is that of the verification of the so-called Penrose proposal [22. Following Friedrich [16, 17, the proposal can be formulated as:

Proposal (Penrose, 1965). Far fields of isolated systems behave like asymptotically simple spacetimes in the sense that they can be smoothly extended to null infinity after a suitable conformal rescaling.

That this proposal is not empty - i.e. that there are examples of radiative spacetimes - is known thanks to work by Chruściel \& Delay $[3$. The idea behind their result was to combine a modification of the gluing construction of Corvino [5], which yields initial data which are Schwarzschildean outside a compact set, with the semiglobal hyperboloidal existence result of Friedrich [12. The resulting spacetime is then - see figure 1-Schwarzschildean in a region, $\widetilde{N}$, of spacetime "near null and spatial infinity". However, at later times radiation could certainly be registered at null infinity. As emphasized by Friedrich, the radiation content of this spacetime is rather special: the Schwarzschildean nature of the initial data implies that Newman-Penrose constants of the spacetime are zero; on the other hand, the Newman-Penrose constants are know

*E-mail address: jav@ap.univie.ac.at 


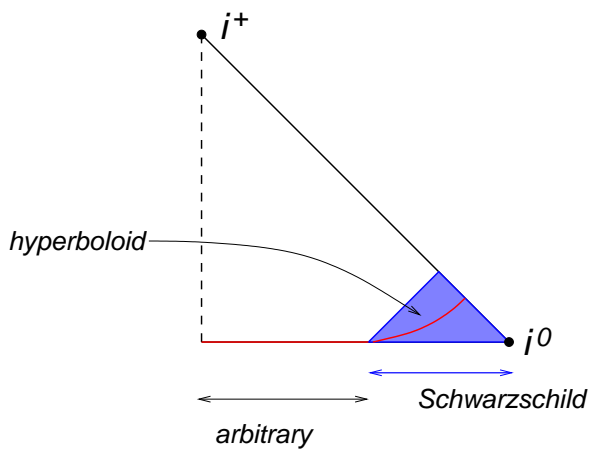

Figure 1: Schematic representation of the asymptotically simple spacetimes constructed by Chruściel \& Delay. The initial data is Schwarzschildean outside a compact set.

to be equal to the value of the rescaled Weyl tensor at timelike infinity $i^{+}$[21, 20]. Hence, the Weyl tensor at $i^{+}$vanishes for the Chruściel-Delay spacetimes, thus implying a fast decaying gravitational field at very late times.

In the light of the results by Chruściel \& Delay it is natural to ask how general can be the behaviour of initial data in a neighbourhood of spatial infinity if one is to obtain asymptotically simple solutions. A first insight to this point was provided by the analysis of Friedrich given in 15. In order to keep the discussions in a reasonable complexity level, his analysis was restricted to time symmetric initial data with an analytic compactification at infinity. He found that a necessary condition in order to attain spacetimes that are smoothly extendible at null infinity is that the regularity condition

$$
D_{\left(a_{s} b_{s}\right.} \cdots D_{a_{1} b_{1}} b_{a b c d)}(i)=0, \quad s=0,1, \ldots,
$$

is satisfied to all orders. If the condition is not satisfied at some order, then the solutions to the Einstein field equations will develop logarithmic singularities at the "sets where null infinity touches spatial infinity". It is worth making a couple of remarks about this condition: firstly, that it is a purely asymptotic condition, and thus it does not pose a big limitation on the kind of physical systems one would like to describe. Secondly, it was proved in [13 that static initial data satisfies the regularity condition.

Recent work by the author [27, using the techniques developed by Friedrich in [15] has shown that the regularity condition (1) is not a sufficient condition to ensure the smoothness of the null infinity arising from developments of the class of initial data under consideration - time symmetric and with an analytic initial 3-metric near infinity. The analysis in [27] considered asymptotic expansions near null and spatial infinity arising from time symmetric data which were further assumed to be conformally flat near infinity. The assumption of conformal flatness near infinity is in this context rather natural because it satisfies automatically the regularity condition (11). The results in [27] lead way to the following conjecture:

Conjecture (Conformally flat data). For every $k>0$ there exists a $p=p(k)$ such that the time development of an asymptotically Euclidean, time symmetric, conformally smooth initial data set which is conformally flat in a neighbourhood $B_{a}(i)$ of infinity admits a conformal extension to null infinity of class $C^{k}$ near spacelike infinity, if and only if the initial data set is Schwarzschildean to order $p(k)$ in $B_{a}(i)$. Moreover, if the conformal extension of the data is smooth — i.e of class $C^{\infty}$ — the data are exactly Schwarzschildean in $B_{a}(i)$.

This conjecture, if found to be true, could be regarded as a rigidity result. It is the objective of the present article to analyse what happens when the assumption of conformal flatness near infinity is removed from the initial data. It could well be the case that the rigidity at spatial infinity suggested by the conjecture is an artifact of the "specialness" of the conformal flatness at infinity. It will be shown that this does not seem to be the case. Our main result - cf. with the main theorem of [27] - is the following. 
Theorem (Main theorem). A necessary condition for the development of time symmetric initial data to be smooth at the intersection of null infinity and spatial infinity is that the data set is at least static to order 3.

What we mean by being static up to an order $p$ will be clarified in the main text. A more technical statement of the theorem will be also be given there. Based on our main theorem, and noting that the only time symmetric data which are conformally flat and static near infinity are the Schwarzschildean ones, we put forward the following conjecture:

Conjecture (General conjecture for time symmetric data). For every $k>0$ there exists a $p=p(k)$ such that the time development of an asymptotically Euclidean, time symmetric data set which is conformally smooth in a neighbourhood $B_{a}(i)$ of infinity admits a conformal extension to null infinity of class $C^{k}$ near spacelike infinity, if and only if the initial data set is static to order $p(k)$ in $B_{a}(i)$. Moreover, if the conformal extension is of class $C^{\infty}$, then the data are exactly static in $B_{a}(i)$.

As stated, the conjecture excludes totally the presence of gravitational radiation near spatial infinity. Whether the latter is a severe limitation for the modeling of systems of physical interest remains to be seen. With regard to non-time symmetric data, one should not expect the things to be any better. In this case it has been shown that, for example, the presence of linear momentum in the initial data produces solutions of the constraints which contain logarithms - see 8. Analogous terms are, in the linear case, source of further non-smoothness at the intersection of null and spatial infinity - see e.g. [25]. In any case, a good picture of the complications of considering non-time symmetric data is not yet available - see however the results given in 28 .

This article is a natural continuation of the investigations undertaken in [15] 19, 26, 27. It also motivates and complements some recent results given in [11.

The article is structured as follows: sections 2 and 3 provide a brief discussion of the so-called regular finite initial value problem near spatial infinity. This discussion is by no means intended to be comprehensive. This material is based on reference [15, to which the reader is remitted for full details. The references [1, 17, 19, 27, 25] may also prove useful. Section 4 considers the construction of initial data satisfying the regularity condition (11), while section 5 discusses the asymptotic expansions near null and spatial infinity obtained from such data. Sections 6 and 7 are devoted to providing an interpretation to the results of section 5 by looking at the static solutions and also to expansions of the Bondi mass near spatial infinity. Finally, section 8 provides some concluding remarks.

\section{General framework}

In this section we review some ideas on the description of the region of spacetime "near null and spatial infinity". Our discussion follows closely that given in [15, and strives to keep its notation and nomenclature as much as possible. The reader is remitted to this reference for the details of the constructions here considered.

\subsection{Spacetime in a neighbourhood of null and spatial infinities}

Let $\left(\widetilde{M}, \widetilde{g}_{\mu \nu}\right)^{1}$ be a vacuum spacetime arising as the development, via the Einstein field equations, of some asymptotically Euclidean initial data $\left(\widetilde{S}, \widetilde{h}_{\alpha \beta}, \widetilde{\chi}_{\alpha \beta}\right)$ with vanishing second fundamental form, $\widetilde{\chi}_{\alpha \beta}=0$-i.e. time symmetric initial data, so that the resulting development has time reflexion symmetry. The metric $\widetilde{h}_{\alpha \beta}$ of the initial hypersurface $\widetilde{S}$ will be taken to be negative definite. For simplicity and definiteness, it will be assumed that $\widetilde{S}$ contains only one asymptotically flat region. It will be further assumed that in this single asymptotically Euclidean region

\footnotetext{
${ }^{1}$ Throughout this work the following conventions will be used. The signature of spacetime metric is $(+,-,-,-)$, thus space metrics are negative definite. The indices $\mu, \nu$ are spacetime ones with range $0, \ldots, 3$, while $\alpha, \beta$ are spatial indices with range $1, \ldots, 3$. Given a 3 dimensional orthonormal frame $e_{(i)}$, the indices $i, j$ denote components with respect to such frame, $i, j=1,2,3$. Finally $a, b, \ldots$ and their primed counterparts $a^{\prime}, b^{\prime}, \ldots$ are spinorial indices taking the values 0,1 .
} 
coordinates $y^{\alpha}$ can be introduced such that

$$
\widetilde{h}_{\alpha \beta}=-\left(1+\frac{2 m}{|y|}\right) \delta_{\alpha \beta}+\mathcal{O}\left(\frac{1}{|y|^{2}}\right),
$$

as $|y| \rightarrow \infty$ and $m$ is the ADM mass of the initial hypersurface.

In order to discuss the behaviour of the development of the initial data sets in the asymptotic region we will make use of the so-called conformal picture. Accordingly, we assume that there is a 3 -dimensional, orientable, smooth compact manifold $(S, h)$ with a point $i \in S$, and a diffeomorphism $\Phi$ of $S \backslash\{i\}$ onto $\widetilde{S}$, and a conformal factor $\Omega$ analytic on $S$ with the properties

$$
\begin{aligned}
& \Omega=0, d \Omega=0, \operatorname{Hess}(\Omega)=-2 h \text { at } i, \\
& \Omega>0 \text { on } S \backslash\{i\}, \\
& h=\Omega^{2} \Phi_{*} \widetilde{h} \text { on } S \backslash\{i\} .
\end{aligned}
$$

It can therefore be checked that under the above conditions

$$
\Phi^{-1}\left(y^{\alpha}\right) \rightarrow i \text { as }|y| \rightarrow \infty,
$$

so that the point $i$ can be rightfully identified with the infinity of the initial hypersurface $\widetilde{S}$. It will be assumed that the conformal factor $\Omega$ is obtained as a result of solving the Einstein constraint equations. For conceptual reasons we shall distinguish the point at infinity $i$ of the initial hypersurface $\widetilde{S}$ from the point $i^{0}$ corresponding to spatial infinity in Penrose's framework.

Now, consider an open ball, $B_{a}(i) \subset S$ of radius $a$ centered on $i$. The radius $a$ is chosen to be so that the open ball is geodesically convex. Let $\rho$ be the geodesic distance along geodesics emanating from $i$. Furthermore, let $\widetilde{N}$ be the domain of influence of the set $\Phi\left(B_{a}(i) \backslash\{i\}\right)$. On intuitive grounds we will refer to $\widetilde{N}$ as being the region of spacetime "close to null and spatial infinities".

It will be convenient for our discussion to blow up the point $i$ representing the infinity of $\widetilde{S}$ to a 2-dimensional sphere. As we are going to make use of a spinorial formalism, the blow up of $i$ is technically achieved by resorting to the bundle $S U(S)$ of normalised spin frames over $S$ with structure group $S U(2, \mathbb{C})$, and projection $\pi$ onto $S$. The details of the blow up will not be given here. The reader is therefore remitted to [15.

It has been shown in [14 that the use of a gauge based on the properties of certain curves known as conformal geodesics renders a conformal factor $\Theta$ that can be read off directly from the initial data, and thus providing an a priori knowledge of the location of the conformal boundary. The conformal factor is given by

$$
\Theta=\kappa^{-1} \Omega\left(1-\tau^{2} \frac{\kappa^{2}}{\omega^{2}}\right)
$$

where

$$
\omega=\frac{2 \Omega}{\sqrt{|h(d \Omega, d \Omega)|}}
$$

and $\kappa$ is an arbitrary function on $S$ such that $\kappa=\rho \kappa^{\prime}$, with $\kappa^{\prime}$ analytic and $\kappa^{\prime}(i)=1$.

Now, let us consider the submanifold $C_{a, \kappa}$ of $\mathbb{R} \times \mathbb{R} \times S U(2, \mathbb{C})$ given by

$$
C_{a, \kappa}=\left\{(\tau, \rho, t) \in \mathbb{R} \times \mathbb{R} \times S U(2, \mathbb{C}) \mid 0 \leq \rho<a,-\frac{\omega}{\kappa} \leq \tau \leq \frac{\omega}{\kappa}\right\} .
$$

Then it can be shown that there is a projection $\pi$ of $C_{a, \kappa}$ onto $\widetilde{N}$ that can be factored as

$$
C_{a, \kappa} \stackrel{\pi_{1}}{\longrightarrow} C_{a, \kappa} / U(1) \stackrel{\pi_{2}}{\longrightarrow} \widetilde{N},
$$

The boundary of $C_{a, \kappa}$ is made of the following submanifolds

$$
\begin{aligned}
& I=\left\{(\tau, \rho, t) \in C_{a, \kappa}|\rho=0, \quad| \tau \mid<1\right\}, \\
& I^{ \pm}=\left\{(\tau, \rho, t) \in C_{a, \kappa} \mid \rho=0, \quad \tau= \pm 1\right\}, \\
& \mathscr{I}^{ \pm}=\left\{(\tau, \rho, t) \in C_{a, \kappa} \mid \rho>0, \quad \tau= \pm \frac{\omega}{\kappa}\right\} .
\end{aligned}
$$




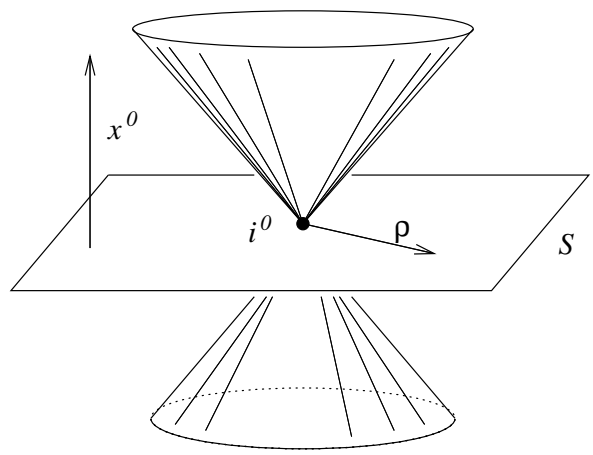

Figure 2: The region of (unphysical) spacetime near spatial and null infinities in the standard representation of spatial infinity as a point. Note that spacetime is the region lying outside the cone passing through $i^{0}$.
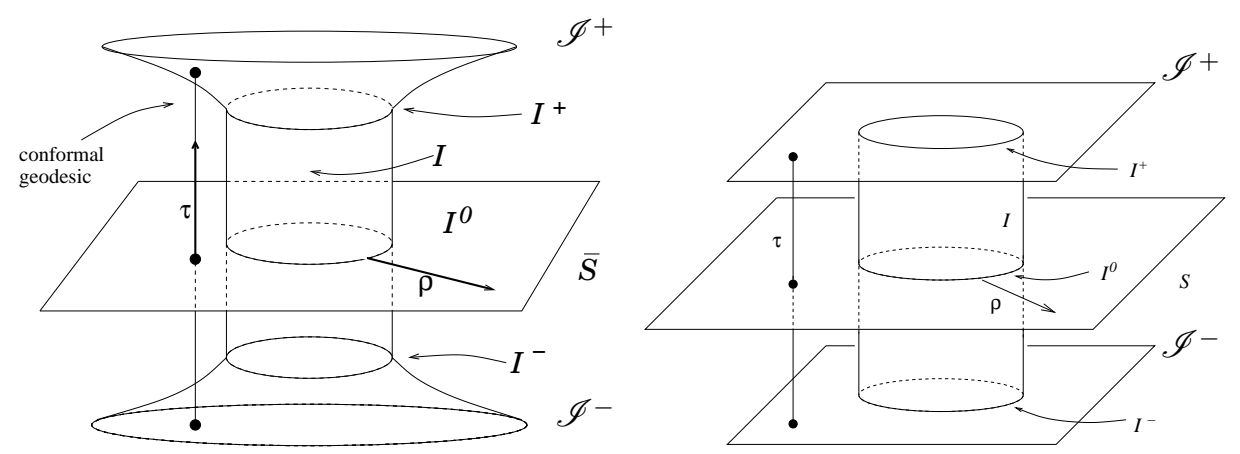

Figure 3: The representation of spatial infinity using the gauge based on the properties of conformal geodesics. The figure to the left corresponds to the choice $\kappa=\rho$; the one to the right corresponds to $\kappa=\omega$ so that the loci of null infinity are hypersurfaces $\tau= \pm 1$.

Calculations in the paradigmatic case of the Minkowski solution show that spacelike geodesics in the physical (unrescaled) spacetime escaping to infinity, map to spatial curves in $C_{a, \kappa}$ terminating at $I$. Similarly null geodesics in the physical spacetime map to geodesics in $C_{a, \kappa}$ with end points at $\mathscr{I}^{ \pm}$- for more details see [25]. This justifies the names of cylinder at spatial infinity for $I$, and of null infinity for $\mathscr{I}^{ \pm}$. The sets $I^{ \pm}$will be considered as neither belonging to $I$ nor to $\mathscr{I}^{ \pm}$. They will be refered to as the sets where spatial infinity "touches" null infinity. The set

$$
\left\{(\tau, \rho, t) \in C_{a, \kappa} \mid \tau=0\right\}
$$

can be, in a natural way with the (compactified) initial hypersurface $S$ once the point at infinity has been blown up.

The function $\kappa$ appearing in the above expressions reflects the remaining bit of conformal freedom available in the framework. It can be used to "fix" the shape of null infinity. Choosing $\kappa=1$ brings us back to the conformal representation where spatial infinity corresponds to a point - see [15, 25] and the figure 2 More interesting for us is to consider a function $\kappa$ of the form $\kappa=\rho \kappa^{\prime}$ where $\kappa^{\prime}$ is an arbitrary positive analytic function on $B_{a}(i)$ such that $\kappa^{\prime}(i)=1$. Here, we will make use of two such choices of $\kappa$. Namely,

$$
\kappa_{1}=\rho, \quad \kappa_{2}=\omega .
$$

The advantage of the first one renders particularly simple analytic expression. The second choice has the peculiarity of yielding an extremely simple formula for the location of null infinity: $\tau= \pm 1$-see figure 3 We will make particular use of this last choice when calculating the Bondi mass near spatial infinity. 


\section{The regular finite initial value problem near spatial in- finity}

The finite representation of the region of spacetime near spatial and null infinity briefly surveyed in the previous section allows the formulation of an initial value problem near spacelike infinity such that: the data and equations are regular; the location and structure of null and spatial is known a priori; and the setting depending on general properties of conformal structures. We refer to this problem as to the regular finite initial value problem near spatial infinity. We now proceed to review some of its features which are relevant for our analysis.

\subsection{The propagation equations}

The propagation equations used by Friedrich in his analysis of the regular finite initial value problem near spatial infinity on [15] are given in a space spinor formalism. This formalism can be thought as the spinorial analogous of the $1+3$ tensorial decompositions. The introduction of such a formalism allows us to work with spinorial quantities having only unprimmed indices. The (timelike) vectorial field on which this decomposition is performed is tangent to certain conformal geodesics. For further details on the space spinor decomposition and the derivation of the propagation equations, the reader is remitted to [23, 14, 15].

The equations given in [15] imply propagation equations for:

(i) the components of the frame $c_{a b}^{\mu}, \mu=0,1, \pm$; the connection coefficients $\Gamma_{a b c d}$ which we decompose as follows

$$
\Gamma_{a b c d}=\frac{1}{\sqrt{2}}\left(\xi_{a b c d}-\chi_{(a b) c d}\right)-\frac{1}{2} \epsilon_{a b} f_{c d} ;
$$

(ii) the Ricci spinor $\Theta_{a b c d}$ which also by convenience is to be decomposed as follows

$$
\Theta_{a b c d}=\Theta_{(a b) c d}-\frac{1}{2} \epsilon_{a b} \Theta_{g}^{g} c d
$$

with $\Theta_{(a b) c d}=\Theta_{(a b)(c d)}$ as $\Theta_{a b c}{ }^{c}=0$;

(iii) the components of the Weyl tensor $\phi_{a b c d}=\phi_{(a b c d)}$, more usually given in terms of the quantities

$$
\phi_{j}=\phi_{(a b c d)_{j}}, \quad j=0, \ldots, 4,
$$

where subindex $j$ in $(a b c d)_{j}$ indicates that after symmetrisation, $j$ indices are to be set equal to 1 .

The propagation equations group naturally in two sets: the equations for what will be known as the $v$-quantities $v=\left(c_{a b}^{\mu}, \xi_{a b c d}, f_{a b}, \chi_{(a b) c d}, \Theta_{(a b) c d}, \Theta_{g c d}^{g}\right), \mu=0,1, \pm$, which are of the form

$$
\partial_{\tau} v=K v+Q(v, v)+L \phi,
$$

where $K$ and $Q$ are respectively linear and quadratic functions with constant coefficients, and $L$ denotes a linear function depending on the coordinates via the functions $\Theta, \partial_{\tau} \Theta$ and a 1-form $d_{a b}$. The linear function $L$ is such that $\left.L\right|_{I}=0$. For a detailed listing of the equations, see [15. 27. Note that the system (5) is essentially a systems of ordinary differential equations for the components of the vector $v$.

The second set of equations is, arguably, the most important part of the propagation equations and corresponds to the evolution equations for the spinor $\phi_{a b c d}$ derived from the Bianchi identities, the Bianchi propagation equations which are of the form

$$
\left(\sqrt{2} E+A^{a b} c_{a b}^{0}\right) \partial_{\tau} \phi+A^{a b} c_{a b}^{1} \partial_{\rho} \phi+A^{a b} c_{a b}^{C} \partial_{C} \phi=B\left(\Gamma_{a b c d}\right) \phi,
$$

where $\phi=\left(\phi_{0}, \phi_{1}, \phi_{2}, \phi_{3}, \phi_{4}\right), \partial_{C}=X_{ \pm}$- see section $4-, E$ denotes the $(5 \times 5)$ unit matrix, $A^{a b} c_{a b}^{\mu}$ are $(5 \times 5)$ matrices depending on the coordinates, and $B\left(\Gamma_{a b c d}\right)$ is a linear matrix valued function of the connection. One has that $\left.\left(A^{a b} c_{a b}^{1}\right)\right|_{I}=0$. 
To the Bianchi propagation equations we add yet another set of three equations, also implied by the Bianchi identities which we refer to as the Bianchi constraint equations. These are of the form

$$
F^{a b} c_{a b}^{0} \partial_{\tau} \phi+F^{a b} c_{a b}^{1} \partial_{\rho} \phi+F^{a b} c_{a b}^{C} \partial_{C}=H\left(\Gamma_{a b c d}\right) \phi,
$$

where $F^{a b} c_{a b}^{\mu}$ denote $(3 \times 5)$ matrices, and $H\left(\Gamma_{a b c d}\right)$ is another matrix valued function of the connection. It turns out that $\left.\left(F^{a b} c_{a b}^{1}\right)\right|_{\tau=0}=0$ which justifies the "constraint" label attached to these equations.

The system of equations (5)-(6) is to be supplemented by initial data which can be constructed as follows

$$
\begin{aligned}
& \Theta_{a b c d}=-\frac{\kappa^{2}}{\Omega} D_{(a b} D_{c d)} \Omega+\frac{1}{12} \kappa r h_{a b c d}, \\
& \phi_{a b c d}=\frac{\kappa^{3}}{\Omega^{2}}\left(D_{(a b} D_{c d)} \Omega+\Omega s_{a b c d}\right), \\
& c_{a b}^{0}=0, \quad c_{a b}^{1}=\kappa x_{a b}, \\
& c^{+}=\kappa\left(\frac{1}{\rho} z_{a b}+\check{c}_{a b}^{+}\right), \quad c^{-}=\kappa\left(\frac{1}{\rho} y_{a b}+\check{c}_{a b}^{-}\right), \\
& \xi_{a b c d}=\sqrt{2}\left\{\kappa\left(\frac{1}{2 \rho}\left(\epsilon_{a c} x_{b d}+\epsilon_{b d} x_{a c}\right)+\check{\gamma}_{a b c d}\right)-\frac{1}{2 \kappa}\left(\epsilon_{a c} D_{b d} \kappa+\epsilon_{b d} D_{a c} \kappa\right)\right\}, \\
& \chi_{(a b) c d}=0, \quad f_{a b}=D_{a b} \kappa .
\end{aligned}
$$

Here, $\Omega$ denotes the conformal factor of the initial hypersurface $S, s_{a b c d}$ the spinorial representation of the symmetric trace free part of the Ricci tensor at the initial hypersurface, $r$ is its Ricci scalar, $D_{a b}$ denotes the covariant derivative on $S$, and $\check{c}_{a b}^{ \pm}$and $\check{\gamma}_{a b c d}$ are the regular parts of the frame and connection on $S$, respectively. The spinors $x_{a b}, y_{a b}, z_{a b}$ and $h_{a b c b d}$ are defined in section 4. Recalling that, $\kappa=\kappa^{\prime} \rho$ with $\kappa^{\prime}(i)=1$, then quantities defined by equations (8a)-88f) are regular for $\rho=0$ if $\Omega, s_{a b c d}$ and $r$ arise from time symmetric initial data with a analytic conformal completion.

\subsection{Transport equations.}

For $p=0,1,2, \ldots$ let $v^{(p)}$ denote the restriction of $\partial_{\rho}^{p} v$ to $I$, that is, $v^{(p)}=\left.\partial_{\rho}^{p} v\right|_{\rho=0}$. Similarly, we write, $\phi^{(p)}=\left.\partial_{\rho}^{p} \phi\right|_{\rho=0}$. For a given integer $p \geq 0$ we will refer to the set of functions $v^{\left(p^{\prime}\right)}$, $0 \leq p^{\prime} \leq p$ as the $s$-jet of order $p$ on $I$, and denote it by $J_{I}^{(p)}(v)$. Similar thing with $J_{I}^{(p)}(\phi)$. If the solutions $v^{(p)}$-or $\phi^{(p)}$ - are evaluated at the intersection of the cylinder $I$ with the initial hypersurface $S$, then we will talk of a $d$-jet of order $p$ on $I^{0}$, and denote it by $J_{I^{0}}^{(p)}(v)$ - or $J_{I^{0}}^{(p)}(\phi)$ respectively. The knowledge of the s-jets, $J_{I}^{(p)}(v)$ and $J_{I}^{(p)}(\phi)$ allows to construct the following Taylor polynomial-like expressions for the vectors $v$ and $\phi$ :

$$
\sum_{k=0}^{p} \frac{1}{k !} v^{(k)} \rho^{k}, \quad \sum_{k=0}^{p} \frac{1}{k !} \phi^{(k)} \rho^{k} .
$$

We will refer to the latter as the order $p$ normal expansions of $v$ and $\phi$. These expansions are to be understood in the sense of truncated series. Our current analytic understanding of the of the propagation equations does not allow us to obtain estimates of the remainders of these expressions. However, it is noted that in the case of linear gravity it has been possible to construct some (non-standard) estimates [18].

The analysis carried out in 25] and continued in the present article makes use of the fact that the cylinder at spatial infinity $I$ is a total characteristic of the propagation equations (5), (6) and (77). This is closely related to the fact that $\left.\left(A^{a b} c_{a b}^{1}\right)\right|_{I}=0$ - see [15) 27] for the details. This general feature of our framework allows us to calculate the vectors $v^{(p)}$ and $\phi^{(p)}$ from a knowledge of the jets $J_{I}^{(p-1)}(v)$ and $J_{I}^{(p-1)}(\phi)$. The equations governing the vectors $v^{(p)}$ and $\phi^{(p)}$ are known 
as the pth order transport equations. These can be written as

$$
\begin{aligned}
\partial_{\tau} v^{(p)}=K v^{(p)}+ & Q\left(v^{(0)}, v^{(p)}\right)+Q\left(v^{(p)}, v^{(0)}\right) \\
& +\sum_{j=1}^{p-1}\left(Q\left(v^{(j)}, v^{(p-j)}\right)+L^{(j)} \phi^{(p-j)}\right)+L^{(p)} \phi^{(0)} .
\end{aligned}
$$

From the Bianchi propagation equations (6) one gets

$$
\begin{aligned}
\left(\sqrt{2} E+A^{a b}\left(c_{a b}^{0}\right)^{(0)}\right) \partial_{\tau} \phi^{(p)}+A^{a b}\left(c_{a b}^{C}\right)^{(0)} \partial_{C} \phi^{(p)}=B\left(\Gamma_{a b c d}^{(0)}\right) \phi^{(p)} & \\
& +\sum_{j=1}^{p}\left(\begin{array}{c}
p \\
j
\end{array}\right)\left(B\left(\Gamma_{a b c d}^{(j)}\right) \phi^{(p-j)}-A^{a b}\left(c_{a b}^{\mu}\right)^{(j)} \partial_{\mu} \phi^{(p-j)}\right) .
\end{aligned}
$$

Similarly, from the Bianchi constraint equations (7) one obtains

$$
\begin{aligned}
F^{a b}\left(c_{a b}^{0}\right)^{(0)} \partial_{\tau} \phi^{(p)} & +F^{a b}\left(c_{a b}^{C}\right)^{(0)} \partial_{C} \phi^{(p)}=H\left(\Gamma_{a b c d}^{(0)}\right) \phi^{(p)} \\
& +\sum_{j=1}^{p}\left(\begin{array}{c}
p \\
j
\end{array}\right)\left(H\left(\Gamma_{a b c d}^{(j)}\right) \phi^{(p-j)}-F^{a b}\left(c_{a b}^{\mu}\right)^{(j)} \partial_{\mu} \phi^{(p-j)}\right) .
\end{aligned}
$$

Diverse properties of the transport equations and how these can be solved are found in [15, 19, 25. In particular in 25] it has been briefly discussed how it is possible to solve these equations by means of computer algebra methods.

\section{Constructing initial fulfilling the regularity condition}

As seen in section 3.1 the initial data for the propagation equations (5), (6) - and consequently also for the transport equations (91)-(10) - can be constructed from a knowledge of the conformal factor $\Omega$, the symmetric and tracefree Ricci spinor $s_{a b c d}$, the Ricci scalar $r$, and the regular part of the frame coefficients and connection, $\check{c}_{a b}^{1}, \check{c}_{a b}^{ \pm}, \check{\gamma}_{a b c d}$-see equations (8a)-8f). Under our assumption of time symmetry, the equation determining $\Omega$ arises from the Hamiltonian constraint by making the so-called conformal Ansatz. The resulting equation is the Licnerowicz equation

$$
\left(\Delta_{h}-\frac{1}{8} r\right)\left(\Omega^{-1 / 2}\right)=4 \pi \delta_{i}
$$

where $\delta_{i}$ is the Dirac-delta function with support on $i$, and $\Delta_{h}$ is the Laplacian with respect to the metric $h$. It is customary to use the following parametrisation for the conformal factor $\Omega$ in a neighbourhood of infinity, $B_{a}(i)$ :

$$
\Omega=\frac{\rho^{2}}{(U+\rho W)^{2}}
$$

where $U$ contains information about the local geometry around $i$, whereas the function $W$ contains information of global nature - e.g. about the ADM mass and higher order multipoles. One has:

$$
\begin{aligned}
& \left(\Delta_{h}-\frac{1}{8} r\right)\left(\frac{U}{\rho^{2}}\right)=4 \pi \delta_{i}, \\
& \left(\Delta_{h}-\frac{1}{8} r\right) W=0,
\end{aligned}
$$

near $i$. Furthermore,

$$
U(i)=1, \quad W(i)=\frac{m}{2} .
$$

Because of the nature of our analysis, we will be just interested in constructing jets $J_{I^{0}}^{(p)}(U)$ and $J_{I^{0}}^{(p)}(W)$ for a certain non-negative integer $p$ consistent with equations (13) and (14). 


\subsection{Freely specifiable data at each order}

In the conformal method to solve the constraint equations, the freely specifiable data under the assumption of time symmetry is given in terms of the conformally rescaled metric $h_{\alpha \beta}$. Due to our coordinate choice $-\rho$ is a geodesic distance - it has the form

$$
h_{\alpha \beta}=\left(\begin{array}{ccc}
1 & 0 & 0 \\
0 & h_{22} & h_{23} \\
0 & h_{23} & h_{33}
\end{array}\right) .
$$

Now, consider an orthonormal frame $e_{(i)}-h_{\alpha \beta} e_{(i)}^{\alpha} e_{(j)}^{\beta}=-\delta_{(i)(j)}$. Associated to the frame $e_{(i)}$ there is a certain spinorial field $c_{a b}$. The correspondence between the two is given by the spatial Infeld-van der Waerden symbols $\sigma_{a b}^{(i)}$, in the form

$$
c_{a b}=\sigma_{a b}^{(i)} e_{(i)} .
$$

Associated to the fields $s_{a b c d}, r, c_{a b}$ on $S$ there are corresponding - $U(1)$ invariant - lifts to the set $\Sigma_{a}=\left\{(\tau, \rho, t) \in C_{a, \kappa} \mid \tau=0\right\}$ which in an abuse of notation we denote by the same symbols. The spinorial field $c_{a b}$ on the initial hypersurface can be written as

$$
c_{a b}=c_{(a b)}=x_{a b} \partial_{\rho}+\left(\frac{1}{\rho} z_{a b}+\check{c}_{a b}^{+}\right) X_{+}+\left(\frac{1}{\rho} y_{a b}+\check{c}_{a b}^{-}\right) X_{-},
$$

where $X_{ \pm}$are differential operators on $S U(2, \mathbb{C})$ related to the $\check{\partial}, \bar{\partial}$ operators of the NP formalism - see [15] for full details. The coefficients $\check{c}_{a b}^{ \pm}$, the regular parts of the frame, satisfy

$$
\check{c}_{a b}^{ \pm}=\mathcal{O}(\rho), \quad \check{c}_{01}^{ \pm}=0 .
$$

The elementary spinors $x_{a b}, y_{a b}, z_{a b}$ are defined as follows

$$
x_{a b}=\sqrt{2} \epsilon_{(a}^{0} \epsilon_{b)}^{1}, \quad y_{a b}=-\frac{1}{\sqrt{2}} \epsilon_{a}{ }^{1} \epsilon_{b}{ }^{1}, \quad z_{a b}=\frac{1}{\sqrt{2}} \epsilon_{a}{ }^{0} \epsilon_{b}{ }^{0} .
$$

We shall also use the spinor

$$
h_{a b c d}=-\epsilon_{a(c} \epsilon_{d) b},
$$

corresponding to the components of the initial metric $h_{\alpha \beta}$ with respect to the spin basis we are using.

The field $c_{a b}$ satisfies the following reality conditions

$$
\check{c}_{00}^{-}=\bar{c}_{11}^{+}, \quad \check{c}_{11}^{-}=\bar{c}_{00}^{+} .
$$

We shall denote the connection associated with the frame $c_{a b}$ by $\gamma_{a b c d}$. Given a spinorial field $\mu_{c d}$, its covarinat derivative is given by

$$
D_{a b} \mu_{c d}=c_{a b}\left(\mu_{c d}\right)-\gamma_{a b c}^{e} \mu_{e d}-\gamma_{a b d}^{e} \mu_{c e} .
$$

We decompose $\gamma_{a b c d}$ into a singular and a regular parts - in concordance with equation [8e as follows

$$
\gamma_{a b c d}=\frac{1}{2 \rho}\left(\epsilon_{a c} \epsilon_{b d}+\epsilon_{b d} \epsilon_{a c}\right)+\check{\gamma}_{a b c d},
$$

where $\check{\gamma}_{a b c d}=\mathcal{O}(\rho)$. The frame coefficients and the connection are related via commutator equations entailing

$$
\begin{aligned}
& \frac{1}{\sqrt{2}} \partial_{\rho}\left(\rho \check{c}_{a a}^{+}\right)=\check{\gamma}_{a a 00}\left(\rho \check{c}_{11}^{+}\right)-\check{\gamma}_{a a 11}\left(\rho \check{c}_{00}^{+}\right)-\frac{1}{\sqrt{2}} \check{\gamma}_{a a 11}, \\
& \frac{1}{\sqrt{2}} \partial_{\rho}\left(\rho \check{c}_{a a}^{-}\right)=\check{\gamma}_{a a 00}\left(\rho \check{c}_{11}^{-}\right)-\check{\gamma}_{a a 11}\left(\rho \check{c}_{00}^{-}\right)-\frac{1}{\sqrt{2}} \check{\gamma}_{a a 00} .
\end{aligned}
$$


Now, the connection coefficients $\check{\gamma}_{a b c d}$ satisfy

$$
\check{\gamma}_{01 c d}=0, \quad \check{\gamma}_{1100}=-\check{\gamma}_{0011}, \quad \check{\gamma}_{a b c d}=\check{\gamma}_{a b d c},
$$

so there are only 5 independent connection coefficients: $\check{\gamma}_{0000}, \check{\gamma}_{0001}, \check{\gamma}_{0011}, \check{\gamma}_{1101}$, and $\check{\gamma}_{1111}$ modulo the reality conditions

$$
\bar{\gamma}_{1101}=\bar{\gamma}_{1110}=\check{\gamma}_{0010}=\check{\gamma}_{0001} \text {. }
$$

The commutator equations (15a)-15b) allow to fully determine the frame coefficients $\check{c}_{a b}^{ \pm}$in terms of the connection coefficients $\check{\gamma}_{a b c d}$. The connection coefficients and the curvature are related via the structure equations of the initial hypersurface. In our case the non-trivial ones can be written as

$$
\begin{aligned}
& \frac{1}{\sqrt{2}} \partial_{\rho} \check{\gamma}_{00 a b}+\frac{1}{\rho}\left\{\check{\gamma}_{0000} z_{a b}-\check{\gamma}_{0011} y_{a b}+\frac{1}{\sqrt{2}} \check{\gamma}_{00 a b}\right\} \\
&=\check{\gamma}_{0000} \check{\gamma}_{11 a b}-\check{\gamma}_{0011} \check{\gamma}_{00 a b}-\frac{1}{2} s_{a b 00}-\frac{1}{6 \sqrt{2}} r y_{a b}, \\
& \frac{1}{\sqrt{2}} \partial_{\rho} \check{\gamma}_{11 a b}+\frac{1}{\rho}\left\{\check{\gamma}_{1100} z_{a b}-\check{\gamma}_{1111} y_{a b}+\frac{1}{\sqrt{2}} \check{\gamma}_{11 a b}\right\} \\
&=\check{\gamma}_{1100} \check{\gamma}_{11 a b}-\check{\gamma}_{1111} \check{\gamma}_{00 a b}-\frac{1}{2} s_{a b 11}-\frac{1}{6 \sqrt{2}} r z_{a b} .
\end{aligned}
$$

The latter, in turn, allow to write the connection coefficients $\check{\gamma}_{a b c d}$ in terms of the traceless Ricci spinor $s_{a b c d}$ and the Ricci scalar $r$. Consequently, also the frame coefficients $c_{a b}^{ \pm}$can be fully written in terms of $s_{a b c d}$ and $r$. The components of $s_{a b c d}$ and $r$ are, however, not independent. They satisfy the 3-dimensional Bianchi identity

$$
D^{a b} s_{a b c d}=\frac{1}{6} D_{c d} r .
$$

Because of its symmetries, the traceless Ricci spinor can be decomposed in terms of elementary spinors as

$$
s_{a b c d}=s_{0} \epsilon_{a b c d}^{0}+s_{1} \epsilon_{a b c d}^{1}+s_{2} \epsilon_{a b c d}^{2}+s_{3} \epsilon_{a b c d}^{3}+s_{4} \epsilon_{a b c d}^{2},
$$

where the coefficients $s_{j}$ are still subject to the reality conditions

$$
s_{4}=\bar{s}_{0}, \quad s_{3}=-\bar{s}_{1}, \quad s_{2}=\bar{s}_{2}
$$

and

$$
\epsilon_{a b c d}^{j}=\epsilon_{(a}\left({ }^{e} \epsilon_{b}{ }^{f} \epsilon_{c}{ }^{g} \epsilon_{d)}{ }^{h)_{j}} .\right.
$$

Details on decomposition of spinors in terms of irreducible terms and on the reality conditions can be found in [15, 19.

In the sequel, it will be convenient to regard the Bianchi identity (17) as providing 3 equations from which the components $s_{1}, s_{2}$ and $s_{3}$ can be determined in terms of the remaining two components $s_{0}, s_{4}$ and the Ricci scalar $r$.

The spinorial field $s_{a b c d}$ and the scalar $r$ are functions on $\mathbb{R} \times S U(2, \mathbb{C})$. Therefore, they admit a decomposition in terms of certain functions, $T_{m}{ }_{k}^{j}, m=0,1,2, \ldots, j, k=0, \ldots, m$, associated with unitary representations of $S U(2, \mathbb{C})$ - the index $m$ being the label of the representation. The functions $T_{m}{ }_{k}^{j}$ are closely related to spin-weighted spherical harmonics via the correspondence - see [19 for details-:

$$
{ }_{s} Y_{n m} \mapsto(-i)^{s+2 n-m} \sqrt{\frac{2 n+1}{4 \pi}} T_{2 n}{ }^{n-m}{ }_{n-s} .
$$

Under the assumptions of time symmetry and analyticity of the conformal metric $h_{\alpha \beta}$ it can be seen - see again [15] for the details - that

$$
\begin{aligned}
& s_{j}=\sum_{p=1}^{\infty} \sum_{q=|2-j|}^{p+1} \sum_{k=0}^{2 q} \frac{1}{p !} s_{j, p ; 2 q, k} T_{2 q}{ }^{k} q-2+j \\
&
\end{aligned}
$$


with $\bar{s}_{j, p ; 2 q, 2 q-k}=(-1)^{k+q} s_{j, p ; 2 q, k}$ and $\bar{r}_{p ; 2 q, 2 q-k}=(-1)^{q+k} r_{p ; 2 q, k}$ as a consequence of the reality conditions. The commutators (15a)-(15b) and the structure equations (16a)-16b) then imply that $\check{c}_{a b}^{ \pm}$and $\check{\gamma}_{a b c d}$ have expansion type $p$. Direct evaluation —using Maple V-up to order $p=6$ shows that,

$$
s_{j, p ; 2 q, k}=s_{j, p ; 2 q, k}\left(s_{j^{\prime}, p^{\prime} ; 2 q^{\prime}, k^{\prime}}, r_{p^{\prime \prime} ; 2 q^{\prime \prime}, k^{\prime \prime}}\right),
$$

where $j=1,2,3, p=1, \ldots, 6, q=|2-j|, \ldots, p, k=0, \ldots, 2 q$ for some $j^{\prime},=0,4,1 \leq p^{\prime} \leq p$, $1 \leq p^{\prime \prime} \leq p,\left|2-j^{\prime}\right| \leq q^{\prime} \leq q, 0 \leq q^{\prime \prime} \leq q$ and $0 \leq k^{\prime} \leq k, 0 \leq k^{\prime \prime} \leq k$. Using (15a)-115b) and (16a)-116b) one can express the coefficients appearing in the expansions for $\check{\gamma}_{a b c d}, \check{c}_{a b}^{ \pm}$in terms of those appearing in (18a) and 18b).

It is worth making a couple of observations. Firstly, the coefficients in the expansions (18a) with $j=0,4$ and in (18b) are not all independent, as these have to be consistent with the expansions of the constraint equations. Another observation is that even if the expansions (18a) and $18 \mathrm{~b}$ ) are already fully consistent with the constraints, some of the coefficients happen to be pure gauge. This is because there is still some conformal freedom left in our construction. How to identify which coefficients in the expansions are gauge and which not will also be discussed in the sequel.

\subsection{Solving the Yamabe equation}

The equation for the function $W$, equation (14), is usually known as the Yamabe equation. As a part of our calculations, it shall be necessary to solve it up to a certain order $p$. This in turn will provide a knowledge of the jet $J_{I}^{(p)}(W)$. If the rescaled metric $h_{\alpha \beta}$ is analytic, then $W$ is also analytic - cfr. 8]. Thus one can write

$$
W=\frac{m}{2}+\sum_{p=1}^{\infty} \frac{1}{p !} w_{p} \rho^{p},
$$

with

$$
w_{p}=\sum_{q=0}^{p} \sum_{k=0}^{2 q} w_{p ; 2 q, k} T_{2 q}{ }^{k},
$$

where the coefficients $w_{p ; 2 q, k}$ are complex numbers satisfying the reality condition $\bar{w}_{p ; 2 q-k}=$ $(-1)^{q+k} w_{p ; 2 q, k}$. Because of the finite order of our calculations, it will not be necessary to assume that $W$ is analytic. However, we will require that it has an 6th-order Taylor polynomial in $\rho$ with coefficients of the form given by equation 19b).

We note that if the initial data is assumed to be conformally flat, then $W$ is harmonic. This implies that

$$
w_{p}=\sum_{k=0}^{2 q} w_{p ; 2 p, k} T_{2 p}{ }_{p}^{k}
$$

If the initial data is not conformally flat, then in the cn-gauge - see $4.4-$ the function $W$ is in general only harmonic up to order $p=3$.

\subsection{The Green function}

The function $U$, solution to equation (13), is also known as the Green function. It can be determined using what is known as the Hadamard's parametrix construction. In this construction one assumes the following Ansatz for the function $U$ :

$$
U=\sum_{p=0}^{\infty} U_{p} \rho^{2 p}
$$

where $U_{p}=U_{p}(\rho, t)$. These can be obtained recursively from

$$
U_{0}=\exp \left\{\frac{1}{4} \int_{0}^{\rho}\left(\Delta_{h} \rho^{2}+6\right) \frac{d s}{s}\right\}, \quad U_{p+1}=-\frac{U_{0}}{(4 p-2) \rho^{p+1}} \int_{0}^{\rho} \frac{\Delta_{h}\left[U_{p}\right] s^{p}}{U_{0}} d s .
$$


In particular, if one has conformally flat data then $U=1$. If $h_{\alpha \beta}$ is analytic near $i$, then the function $U$ is analytic, and furthermore, the coefficients $U_{p}$ are also analytic.

\subsection{The cn-gauge}

The construction of the functions $W$ and $U$ described before does not fix the functions uniquely. A rescaling of the form

$$
h \rightarrow h^{\prime}=\vartheta^{4} h, \quad \Omega \rightarrow \Omega^{\prime}=\vartheta^{2} \Omega,
$$

with a conformal factor $\vartheta$ satisfying $\vartheta(i)=1$, leaves $\widetilde{h}=\Omega^{-2} h$ unchanged, but implies

$$
U \rightarrow U^{\prime}=\frac{\rho^{\prime}}{\rho} \vartheta^{-1}, \quad W \rightarrow W^{\prime}=\vartheta^{-1} W,
$$

where $\rho^{\prime}$ is the $h^{\prime}$ geodesic distance along geodesics starting at $i$. In order to remove this freedom our discussion will done in a certain gauge known as the cn-gauge. Note however, that our whole discussion could have been carried in another gauge - for example, the discussion in [11] uses a certain gauge for which $W=m / 2$.

The cn-gauge is defined as follows. Consider the 3-dimensional conformal geodesic equations

$$
\begin{aligned}
& \dot{x}^{\beta} \nabla_{\beta} \dot{x}^{\alpha}=-2 b_{\beta} \dot{x}^{\beta}+\dot{x}_{\beta} \dot{x}^{\beta} b^{\alpha}, \\
& \dot{x}^{\beta} \nabla_{\beta} b_{\alpha}=b_{\beta} \dot{x}^{\beta} b_{\alpha}-\frac{1}{2} b_{\beta} b^{\beta} \dot{x}_{\alpha}+\left(s_{\alpha \beta}+\frac{1}{12} r h_{\alpha \beta}\right) \dot{x}^{\beta},
\end{aligned}
$$

where now $x^{\alpha}(t)$ is a curve on $S$, and $b^{\alpha}$ an associated 3-dimensional 1-form. Indices are raised and lowered using $h_{\alpha \beta}$ and $h^{\alpha \beta}$ respectively. We supplement the latter equations with the initial conditions

$$
x(0)=i, \quad \dot{x}_{\beta} \dot{x}^{\beta}=-1, \quad b(0)=0 .
$$

It is not hard to see that if $a$ is chosen small enough, there exists a unique solution to these equations on $B_{a}(i)$. Furthermore, there exist in $B_{a}(i)$ a unique rescaling of the form (20a)-(20b) such that

$$
b_{\beta} \dot{x}^{\beta}=0 \quad \text { on } B_{a}(i)
$$

can always be attained. A metric in the conformal class for which the condition (23) is satisfied along the solutions of the 3-dimensional conformal geodesic equations (21) and (22) will be said to be in the cn-gauge.

It is noticed that for conformally flat data, if $\dot{x}$ is the tangent to (standard) geodesics starting at $i$ with $\dot{x}_{\beta} \dot{x}^{\beta}=-1$, and one requires $b \equiv 0$ in $B_{a}(i)$, one is already in the cn-gauge. This is the reason why the calculations discussed in [9, 26, 27, did not contemplate this point.

The cn-gauge condition (23) imposes restrictions on $s_{\alpha \beta}$-and consequently in its spinorial representation $s_{a b c d}$ - and $r$. One can obtain a space-spinor version of the conformal geodesic equations (21) and (22) by contracting with the frame $e_{(i)}$, and then using the spatial Infeld van der Waerden symbols. These spinorial equations together with the results of $\S 4.1$ yield after some calculations in Maple $\mathrm{V}$ the following result.

Lemma 1 (the free specifiable data in the cn-gauge). In the cn-gauge one has that

$$
s_{j, 1 ; 2 q, k}=0, \quad r_{1 ; 2 q, k}=0,
$$

for $j=0, \ldots, 4, q=0, \ldots, 2, k=0, \ldots, 2 q$. Furthermore,

$$
s_{j, p ; 2(p+1), k}=0,
$$

for $j=0, \ldots, 4, p=2, \ldots, 6, k=0, \ldots, 2 q$. The coefficients of the expansion of the trace free Ricci spinor and the Ricci scalar are of the form

$$
\begin{aligned}
& s_{j, p ; 2 q, k}=s_{j, p ; 2 q, k}\left(s_{0, p ; 2 q, k}, s_{4, p ; 2 q, k}\right), \\
& r_{p ; 2 q, k}=r_{p ; 2 q, k}\left(s_{0, p ; 2 q, k}, s_{4, p ; 2 q, k}\right),
\end{aligned}
$$

for $j=1,2,3$ and $p=2, \ldots, 6, q=2, \ldots, p, k=0, \ldots, 2 q$.

It is stressed once more that the results are valid for expansions up to order $p=6$ - the order up to which the Maple V calculations have been carried out. 


\subsection{Fulfilling the regularity condition}

We begin by recalling the following important result by Friedrich [15].

Theorem 1 (Friedrich,1998). The solutions to the transport equations are smooth through $I^{ \pm}$ only if the (regularity) condition

$$
D_{\left(a_{s} b_{s}\right.} \cdots D_{a_{1} b_{1}} b_{a b c d)}(i)=0, \quad s=0,1, \ldots
$$

is satisfied by the free initial data.

As discussed in extensis in 27] the great simplification of studying expansions for conformally flat data lies in the fact that these satisfy the regularity condition (1) trivially. In the current analysis the situation is completely different. Given free data in the cn-gauge - in the way discussed in lemma 1- one still has specialise to those free specifiable data consistent with the regularity condition. The question is now, how to implement the regularity condition? A first - naive - approach would be to calculate directly the spinor $b_{a b c d}$ and then its symmetrised derivatives up to the required order. This approach is computationally too involved, so we have opted for a different approach. In 15 it has been shown that the if the regularity condition holds up to a certain order, then what is known as the massless part of the Weyl tensor has a particular form. We shall make use of this result.

The massless part of the Weyl tensor is given by

$$
\begin{aligned}
\phi_{a b c d}^{\prime}=\frac{1}{\rho^{4}} & \left(U^{2} D_{(a b} D_{c d)} \rho^{2}-4 U D_{(a b} \rho^{2} D_{c d)} U\right. \\
& \left.\quad-2 \rho^{2} U D_{(a b} D_{c d)} U+6 \rho^{2} D_{(a b} U D_{c d)} U+\rho^{2} U^{2} s_{a b c d}\right) .
\end{aligned}
$$

Let also

$$
\breve{\phi}_{a b c d}^{\prime}=\kappa^{3} \phi_{a b c d}^{\prime}
$$

The spinor $\breve{\phi}_{a b c d}^{\prime}$ can be seen to have an expansion of the form

$$
\breve{\phi}_{j}^{\prime}=\sum_{p=|2-j|}^{\infty} \sum_{q=|2-j|}^{p} \sum_{k=0}^{2 q} \frac{1}{p !} \breve{\phi}_{j, p ; 2 q, k}^{\prime} T_{2 q}{ }^{k}{ }_{q-2+j} \rho^{p},
$$

where $\breve{\phi}_{j}^{\prime}=\breve{\phi}_{(a b c d)_{j}}^{\prime}$. Using the aforedefined quantities, one has the following lemma.

Lemma 2. The following two conditions are equivalent:

i)

$$
D_{\left(a_{q} b_{q}\right.} \cdots D_{a_{1} b_{1}} b_{a b c d)}(i)=0, \quad q=0,1, \ldots, s
$$

ii)

$$
\breve{\phi}_{j, p, 2 p, k}^{\prime}=0, \quad p=0,1, \ldots, s, \quad k=0, \ldots, 2 p, \quad j=0, \ldots, 4 .
$$

The proof of lemma 2 can be found in reference [15]. From lemma 2] direct calculations using Maple $\mathrm{V}$ lead to the following result.

Lemma 3. In the cn-gauge, if the regularity condition (1) holds up to order $s=4$, then

$$
s_{j}=\sum_{p=2}^{6} \sum_{k=0}^{2 p} \frac{1}{p !} s_{j, p ; 2 p, k} T_{2 p}{ }^{k}{ }_{p+j-2} \rho^{p}+\cdots,
$$

with $j=0, \ldots, 4$. Furthermore,

$$
r=\sum_{p=2}^{6} \sum_{k=0}^{2 p} \frac{1}{p !} r_{p ; 2 p, k} T_{2 p}{ }^{k} \rho^{p}+\cdots
$$

This last result would seem to indicate that $r$ is an harmonic function. Explicit calculations up to order $p=7$ show that this is not the case. 


\subsection{Initial data for the propagation equations}

Starting from lemma 3 and taking the discussion of section 4 backwards, one can calculate d-jets $J_{I^{0}}^{(6)}(v)$ and $J_{I^{0}}^{(6)}(\phi)$ consistent with the regularity condition (1). We summarise this construction: start with given $s_{0}$ and $s_{4}\left(s_{4}=\bar{s}_{0}\right)$, components of the spinor $s_{a b c d}$ which in the cn-gauge are of the form

$$
\begin{aligned}
& s_{0}=\sum_{p=2}^{6} \sum_{k=0}^{2 p} \frac{1}{p !} s_{0, p ; 2 p, k} T_{2 p}{ }^{k}{ }_{p-2} \rho^{p}+\cdots, \\
& s_{4}=\sum_{p=2}^{6} \sum_{k=0}^{2 p} \frac{1}{p !} s_{4, p ; 2 p, k} T_{2 p}{ }^{k}{ }_{p+2} \rho^{p}+\cdots .
\end{aligned}
$$

Using the Bianchi identities one can calculate the remaining components $s_{1}, s_{2}, s_{3}$, and because of the cn-gauge, also the Ricci scalar $r$. Now, using the structure equations (16a)-(16b) first, and then the commutator equations (15a)-15b) one can calculate the regular part of the connection and the frame, $\check{\gamma}_{a b c d}, \check{c}_{a b}^{ \pm}$up to order 6 inclusive. Using these, one can construct a function $W$ consistent up to order 6 with the help of equation (14). Similarly, using Hadamard's procedure one can calculate the function $U$ up to order 7 . The latter requires the evaluation of the steps 0,1,2 of Hadamard's recursive procedure. Because of lemma [3] such $U$ yields a massless part of the Weyl tensor such that the regularity condition (1) is satisfied to order 6. Knowledge of $W$ to order 6 and $U$ to order 7 allows to calculate the conformal factor $\Omega$ to order 9 inclusive. This is exactly what is required in order to calculate the d-jets, $J_{I^{0}}^{(6)}(v)$ and $J_{I^{0}}^{(6)}(\phi)$. These by construction should be consistent with the regularity condition (11).

A final result ensures that our whole construction of initial data for the conformal propagation equations is consistent.

Lemma 4 (fulfillment of the Bianchi constraint equations). Assume that the components $s_{0}$ and $s_{4}$ of the Ricci spinor are of the form given by 24) and 25). Further, assume that the connection and frame coefficients $\check{\gamma}_{a b c d}$ and $\check{c}_{a b}$ have been calculated up to order 6 using the structure and commutator equations. Then, the Bianchi constraint transport equations (7) at $\tau=0$ are satisfied up to order 6.

The proof of lemma 4 proceeds, again, by direct evaluation.

\section{Solving the transport equations}

The d-jets $J_{I^{0}}^{(6)}(v)$ and $J_{I^{0}}^{(6)}(\phi)$ described in section 4.6 provide initial data for the transport equations of order $p=1, \ldots, 6$. Their solution would in turn yield the s-jets $J_{I}^{(6)}(v)$ and $J_{I}^{(6)}(\phi)$ and thus the vector unknowns $v$ and $\phi$ up to order 6 . Before describing these solutions, a list of the assumptions being made is given.

Assumptions. In order to calculate the s-jets $J_{I}^{(6)}(v)$ and $J_{I}^{(6)}(\phi)$ it has been assumed that:

(i) one has a time symmetric initial data set with an analytic conformal metric $h_{\alpha \beta}$ - respect to normal coordinates - in a ball $B_{a}(i)$ of infinity;

(ii) the initial data for the conformal propagation equations has been calculated in the cn-gauge in the way described in section 4.6 .

(iii) the following choices for the function $\kappa$ appearing in the conformal factor $\Theta$ have been used:

$$
\kappa=\rho, \quad \kappa=\omega
$$

Remark. It is noted that the assumption of analyticity of the conformal compactification of the initial hypersurface is not strictly needed. It is given here so for simplicity. It can be 
conveniently substituted by the assumption that certain functions have Taylor polynomials of a given order with decompositions in spherical harmonics consistent with the expansion types.

The procedure to solve the transport equations (9) and (10) is extremely lenghty, but nevertheless straightforward. Thus, it is quite amenable to a computer algebra treatment. A "transport equations solver" script in the computer algebra system Maple V has been implemented for the calculations described in 27. The same scripts can be used, without the need of further modifications, to perform the calculations required for the present article. Because of the length of the expressions obtained, only qualitative features of the solutions obtained are given. A detailed description of the computer algebra implementation will be described elsewhere.

Our first result is the following:

Theorem 2 (solutions up to order 4). Under the assumptions (i)-(iii), the solutions of the transport equations for $p=0, \ldots, 4$ are polynomial in $\tau$. Thus, they extend smoothly to the sets $I^{ \pm}$

Remark 1. This last theorem is independent of the choice of the function $\kappa$. The polynomial expressions involved will nevertheless be different for each choice, but of the order of the polynomials is the same.

Remark 2. A complete listing of the solutions to the transport equations up to order 3 with the choice $\kappa=\rho$ is given in [19. A description of the solutions of the Bianchi transport equations for conformally flat data up to order 4 with $\kappa=\rho$ are given in [27].

As it is to be expected from the results of [27, the situation is different when considering solutions to the transport equations with $p \geq 5$.

Theorem 3 (obstructions at order 5). Under the assumptions (i)-(iii), the solutions to the $v$-transport equations (9) at order $p=5$ are polynomial in $\tau$ and therefore smooth. On the other hand, the solutions to the $p=5$ Bianchi transport equations (10) are of the form

$$
\phi_{j}^{(5)}=\Upsilon^{(5)}\left(f_{j}(\tau) \ln (1-\tau)+g_{j}(\tau) \ln (1+\tau)\right)+h_{j}(\tau),
$$

for $j=0, \ldots, 4$, where $f_{j}(\tau), g_{j}(\tau)$ and $h_{j}(\tau)$ are polynomials in $\tau$. The polynomials $f_{j}(\tau), g_{j}(\tau)$ are all of order 7 . In particular

$$
f_{4}(\tau)=g_{0}(-\tau)=(1-\tau)^{3} k(\tau)
$$

where $k(0) \neq 0$. Furthermore

$$
\Upsilon^{(5)}=\sum_{k=0}^{4} \Upsilon_{k}^{(5)} T_{4}{ }_{2}^{k}
$$

with

$$
\begin{aligned}
& \Upsilon_{0}^{(5)}=18 m w_{2 ; 4,0}-36 \sqrt{6} w_{1 ; 2,0}^{2}+r_{2 ; 4,0}, \\
& \Upsilon_{1}^{(5)}=18 m w_{2 ; 4,0}-72 \sqrt{3} w_{1 ; 2,0} w_{1,2,1}+r_{2 ; 4,1}, \\
& \Upsilon_{2}^{(5)}=18 m w_{2 ; 4,2}-72 w_{1 ; 2,1}^{2}-72 w_{1 ; 2,0} w_{1 ; 2,2}+r_{2 ; 4,2}, \\
& \Upsilon_{3}^{(5)}=18 m w_{2 ; 4,3}-72 \sqrt{3} w_{1 ; 2,1} w_{1 ; 2,2}+r_{2 ; 4,3}, \\
& \Upsilon_{4}^{(5)}=18 m w_{2 ; 4,4}-36 \sqrt{6} w_{1 ; 2,2}^{2}+r_{2 ; 4,4} .
\end{aligned}
$$

Thus, as a consequence of this theorem, the solutions of the $p=5$ Bianchi transport equations are - in general - not smooth (i.e. $C^{\infty}$ ), but merely of class $C^{2}$ at the sets $I^{ \pm}$. Because of this, the quantities $\Upsilon_{j}^{(5)}, j=0, \ldots, 4$ will be referred as to the order 5 obstructions. There are several remarks that come now into place.

Remark 1. Firstly, note that if one sets $r_{2,4, k}=0, k=0, \ldots 4$ one recovers the order 5 obstructions found in [27] for conformally flat data.

Remark 2. For conformally flat initial data the obstructions coincide - modulo some irrelevant numerical factor- with the Newman-Penrose constants of the time development. For the 
developments of non-conformally flat time symmetric data the Newman-Penrose constants can be expressed in terms of the initial data by

$$
\begin{aligned}
& G_{0}^{(5)}=18 m w_{2 ; 4,0}-36 \sqrt{6} w_{1 ; 2,0}^{2}-\frac{1}{1016} r_{2 ; 4,0}, \\
& G_{1}^{(5)}=18 m w_{2 ; 4,0}-72 \sqrt{3} w_{1 ; 2,0} w_{1,2,1}-\frac{1}{1016} r_{2 ; 4,1}, \\
& G_{2}^{(5)}=18 m w_{2 ; 4,2}-72 w_{1 ; 2,1}^{2}-72 w_{1 ; 2,0} w_{1 ; 2,2}-\frac{1}{1016} r_{2 ; 4,2}, \\
& G_{3}^{(5)}=18 m w_{2 ; 4,3}-72 \sqrt{3} w_{1 ; 2,1} w_{1 ; 2,2}-\frac{1}{1016} r_{2 ; 4,3}, \\
& G_{4}^{(5)}=18 m w_{2 ; 4,4}-36 \sqrt{6} w_{1 ; 2,2}^{2}-\frac{1}{1016} r_{2 ; 4,4} .
\end{aligned}
$$

Thus, we see that in general for time symmetric constants the order 5 obstructions are different to the Newman-Penrose constants of the time development. The coincidence occurring in the case of conformally flat data lead to conjecture that because static solutions should have no logarithmic terms in their expansions then their Newman-Penrose constants should be zero. Our results show that this is also in general not the case. Explicit calculations using the Weyl class of solutions by S. Dain have lead to the same conclusion [6].

Remark 3. From the way lower order solutions to the transport equations feed into higher order transport equations that if the obstructions (26)-(30) do not vanish then the solutions $v^{(6)}$ will already contain terms of the form $\ln (1 \pm \tau)$, and even more, $\phi^{(6)}$ will contain $\ln ^{2}(1 \pm \tau)$ terms. The situation is bound to be even worse for $p \geq 7$. The hyperbolic nature of the propagation equations implies in turn that the non-smoothness of the solutions of the transport equations at $I^{ \pm}$will be propagated along the generators of null infinity. However, a proper discussion of this phenomenon is still to be made.

Our investigation is focused on solutions to the transport equations which are consistent with a time development of the initial with smooth null infinity. Therefore, the situation of most interest for us is when the so-called order 5 obstructions vanish. The consideration of order 6 transport equations under this assumption leads to the following result.

Theorem 4 (obstructions at order 6). Under the assumptions (i)-(iii) and if the order 5 obstructions (26)-(30) vanish, the solutions to the v-transport equations (9) at order $p=6$ are polynomial in $\tau$. The solutions to the $p=6$ Bianchi transport equations (10) are of the form

$$
\phi_{j}^{(6)}=\Upsilon^{(6)}\left(\hat{f}_{j}(\tau) \ln (1-\tau)+\hat{g}_{j}(\tau) \ln (1+\tau)\right)+\hat{h}_{j}(\tau),
$$

for $j=0, \ldots, 4$, where $\hat{f}_{j}(\tau), \hat{g}_{j}(\tau)$ and $\hat{h}_{j}(\tau)$ are again polynomials in $\tau$. The polynomials $\hat{f}_{j}(\tau)$, $\hat{g}_{j}(\tau)$ are of order 9 , and in particular

$$
\hat{f}_{4}(\tau)=\hat{g}_{0}(-\tau)=(1-\tau)^{4} \hat{k}(\tau),
$$

where $\hat{k}(0) \neq 0$. Furthermore

$$
\Upsilon^{(6)}=\sum_{k=0}^{6} \Upsilon_{k}^{(6)} T_{6}{ }_{3}^{k}
$$

with

$$
\begin{aligned}
& \Upsilon_{0}^{(6)}=24 m^{2} w_{3 ; 6,0}-48 \sqrt{15} m w_{1 ; 2,0} w_{2 ; 4,0}+r_{3 ; 6,0} \\
& \Upsilon_{1}^{(6)}=24 m^{2} w_{3 ; 6,1}-48 \sqrt{10} m w_{1 ; 2,0} w_{2 ; 4,1}-48 \sqrt{5} m w_{1 ; 2,1} w_{2 ; 4,0}+r_{3 ; 6,1}, \\
& \Upsilon_{2}^{(6)}=24 m^{2} w_{3 ; 6,2}-72 \sqrt{6} m w_{1 ; 2,0} w_{2 ; 4,2}-72 \sqrt{2} m w_{1 ; 2,1} w_{2 ; 4,1}+r_{3 ; 6,2}, \\
& \Upsilon_{3}^{(6)}=24 m^{2} w_{3 ; 6,3}-144 m w_{2 ; 4,2} w_{1 ; 2,1}-48 \sqrt{3} m w_{1 ; 2,0} w_{2 ; 4,3}-48 \sqrt{3} m w_{1 ; 2,2} w_{2 ; 4,1}+r_{3 ; 6,3}, \\
& \Upsilon_{4}^{(6)}=24 m^{2} w_{3 ; 6,4}-72 m \sqrt{6} w_{1 ; 2,2} w_{2 ; 4,2}-72 \sqrt{2} m w_{1 ; 2,1} w_{2 ; 4,3}+r_{3 ; 6,4}, \\
& \Upsilon_{5}^{(6)}=24 m^{2} w_{3 ; 6,5}-48 \sqrt{10} m w_{1 ; 2,2} w_{2 ; 4,3}-48 \sqrt{5} m w_{1 ; 2,1} w_{2 ; 4,4}+r_{3 ; 6,5}, \\
& \Upsilon_{6}^{(6)}=24 m^{2} w_{3 ; 6,6}-48 \sqrt{15} m w_{1 ; 2,2} w_{2 ; 4,4}+r_{3 ; 6,6}
\end{aligned}
$$


Again, if one sets the terms associated with the Ricci scalar, $r_{2 ; 4, k}, r_{3 ; 6, k}$ to zero, one recovers the obstructions obtained in 27] for conformally flat initial data.

It is mentioned by passing that it is quite likely that the order 5 and order 6 obstructions (26)(30) and (31)-(37) are associated to some kind of conserved quantities at null infinity. Using an analysis along the lines of the asymptotic characteristic initial value problem, it was shown in 4] that there are certain conserved quantities — besides the so-called logarithmic Newman-Penrose constants - associated with the first logarithmic terms appearing in their expansions. In the basis of our results we speculate that these conserved constants are related with our obstructions, although a discussion of this will also be left for the future.

The complexity of the expressions involved preclude us from a direct evaluation of the order 7 and higher solutions to the transport equations. In order to reduce this complexity one can consider data with axial symmetry. In this way it is possible to complete the expansions up to order 8 inclusive. Due to the axial symmetry, there is only one obstruction at every order. These are:

$$
\begin{aligned}
& \Upsilon_{4}^{(7)}=5040 m w_{4 ; 8,4}-23040 w_{1 ; 2,1} w_{3 ; 6,3}-25920 w_{2 ; 4,2}^{2}-161 r_{4 ; 8,4} \\
& \Upsilon_{5}^{(8)}=1440 m w_{5 ; 10,5}-4800 w_{1 ; 2,1} w_{4 ; 8,4}-19200 w_{2 ; 4,2} w_{3 ; 6,3}+37 r_{5 ; 10,5}
\end{aligned}
$$

\section{The obstructions and the static initial data}

In order to provide an interpretation of the obstructions obtained in the previous section, and in view of the results of [27], let us consider for a moment static initial data given in the cn-gauge. The static data gives rise to time developments with a smooth null infinity [7. Thus, one should expect the obstructions should automatically vanish for this kind of data.

The line element of static solutions to the field equations can be written, in the physical spacetime in the form

$$
\widetilde{g}=\psi^{2} d t^{2}+\widetilde{h}
$$

where $\widetilde{h}$ is a $t$-independent, negative definite, 3 -metric, and $\psi$ is its $t$-independent norm, $\psi=$ $\sqrt{\widetilde{h}(k, k)}$ of the Killing vector field $k=\partial_{t}$. The static vacuum field equations are then given by

$$
\begin{aligned}
& \widetilde{r}_{\alpha \beta}=\frac{1}{\psi} \widetilde{D}_{\alpha} \widetilde{D}_{\beta} \psi, \\
& \Delta_{\widetilde{h}} \psi=0,
\end{aligned}
$$

where $\widetilde{r}_{\alpha \beta}, \widetilde{D}, \Delta_{\widetilde{h}}$ are respectively the Ricci tensor, the covariant derivative and the Laplacian with respect to the metric $\widetilde{h}$. Now, introducing the rescaling $h_{\alpha \beta}=\Omega^{2} \widetilde{h}_{\alpha \beta}$ where $\Omega$ is the conformal factor arising from the Licnerowicz equation (12), contracting with the frame $e_{(i)}, i=1,2,3$ and then using the spatial Infeld symbols $\sigma_{a b}^{(i)}$ one arrives to the following (space spinorial) form of the static equations

$$
\begin{gathered}
s_{a b c d}+\frac{1}{3} r h_{a b c d}+\frac{1}{\Omega} D_{a b} D_{c d} \Omega+h_{a b c d}\left(\frac{1}{\Omega} \Delta_{h} \Omega-\frac{2}{\Omega^{2}} D^{e f} D_{e f} \Omega\right) \\
=\frac{1}{\psi}\left(D_{a b} D_{c d} \psi+\frac{2}{\Omega} D_{(a b} D_{c d)} \Omega-\frac{1}{\Omega} h_{a b c d} D^{e f} \psi D_{e f} \Omega\right), \\
\Delta_{h}\left(\Omega^{-1 / 2} \psi\right)=\frac{1}{8} r \Omega^{-1 / 2} \psi .
\end{gathered}
$$

Given a time symmetric initial data set in a the cn-gauge - and following previous discussionswe will say that the initial data set is static up to order $p$ if given the corresponding order $p$ d-jets $J_{I^{0}}^{(p)}(v), J_{I^{0}}^{(p)}(\phi)$, there is a static solution with order $p$ d-jets $J_{I^{0}}^{(p)}\left(v_{s}\right), J_{I^{0}}^{(p)}\left(\phi_{s}\right)$ such that

$$
J_{I^{0}}^{(p)}(v)=J_{I^{0}}^{(p)}\left(v_{s}\right), \quad J_{I^{0}}^{(p)}(\phi)=J_{I^{0}}^{(p)}\left(\phi_{s}\right) .
$$

Substituting the expansions for $s_{a b c d}, \Omega, r$ and the connection and frame associated with $D_{a b}$ found in section $\S 4$, one obtains after some Maple $\mathrm{V}$ calculations the following lemma. 
Lemma 5. Given the assumptions (i)-(iii), necessary and sufficient conditions for a time symmetric initial data set to be static up to order 3 are that the obstructions (26)-(30) and (31)- (37) vanish.

Combining theorems 3 and 4 together with the previous lemma, we have the proved our main result, and thus provided an interpretation of the obstructions (26)-(30) and (31)-(37).

Theorem (Main theorem, technical version). Necessary condition for the s-jets $J_{I}^{(7)}(v)$ and $J_{I}^{(6)}(\phi)$ to extend smoothly to the sets $I^{ \pm}$is that the initial data set is static up to order 3.

Higher order results along the same lines can be obtained if one restricts the analysis to initial data which is axially symmetric. All together, these results provide what we believe is fair evidence for the general conjecture for time symmetric data put forward in the introduction.

\section{Expansions for the Bondi mass near spatial infinity}

It is of interest to see how the results of the previous section can be obtain by means of a different kind of arguments. Namely, we will show how one can relate the obstructions obtained in the previous sections to the Bondi mass. It is intuitively clear - although its verification requires some calculations, see e.g. [11] - that if one starts with a Cauchy problem near spatial infinity as the one described here, in which the initial data is static, the Bondi mass should be constant and equal to the ADM mass of the spacetime. The converse, namely starting from a regular finite Cauchy problem at spatial infinity with the additional requirement that the Bondi mass should be constant to deduce the existence of a static Killing vector near spatial infinity, is also intuitively plausible but much more complicated, requiring an existence theorem for the Cauchy problem in the neighbourhood of the sets $I^{ \pm}$which is not yet available.

The standard way of introducing the Bondi mass and other quantities defined at null infinity - like the Newman-Penrose constants - is to construct an ad hoc coordinate system and frame well adapted to the geometry of spacetime near the intersection of null infinity with an outgoing light cone. We will refer to these coordinates and the concomitant adapted frame to as the Newman-Penrose (NP) gauge. A detailed discussion on how to construct this gauge can be found in [19. In the NP gauge - and using the NP formalism notation- the Bondi mass is given by

$$
m_{B}=-\frac{1}{4 \pi} \oint\left(\psi_{2}^{0}-\sigma_{0} \dot{\bar{\sigma}}_{0}\right) d S
$$

where the functions $\psi_{2}^{0}$ and $\sigma_{0}$ - depending on a retarded time $u$ and the angular dependenceare the leading terms of the component $\widetilde{\psi}_{2}$ of the Weyl tensor and the spin coefficient $\sigma$ - the shear. These quantities are given in the physical space time, and for the class of solutions of the Einstein equations under consideration it can be seen that

$$
\widetilde{\psi_{2}}=\psi_{2}^{0} \Theta^{3}+\cdots, \quad \widetilde{\sigma}=\sigma_{0} \Theta^{2}+\cdots .
$$

The integral in (38) is evaluated over the cut of $\mathscr{I}^{+}$defined by $u=$ const. The relevance of the Bondi mass is that under suitable assumptions it can be shown to be non-negative and nonincreasing, i.e. $\dot{m}_{B}=\partial_{u} m_{B}=0$. Furthermore, under some extra assumptions it can be shown to tend to the ADM mass of the spacetime as one approaches spatial infinity — see 1, 19, 26. It is worth pointing that all these discussions about the limit of the Bondi mass contain fide assumptions that shall eventually be removed once a complete theory of spatial infinity is achieved.

A major inconvenient of the Bondi mass in the form given by formula (38) is the gauge in which it is given, which although very convenient for theoretical discussions, hampers its evaluation in direct examples. The connection between the NP gauge and the gauge used in the present article - sometimes refered as to the $\mathrm{F}$ gauge - has been obtained in 19. The transformation between gauges involves the determination of a conformal factor $\theta$, such that $\theta \Theta$ is the conformal factor in the NP gauge, and a rotation of the frame $c_{a a^{\prime}}$ introduced in section 2, and which is realized by matrices $\Lambda_{b}^{a} \in S L(2, \mathbb{C})$. Under the choice, $\kappa=\omega$, of the function $\kappa$ appearing in the conformal 
factor given in equation(2), the locus of null infinity is given in a simple way by $\tau= \pm 1$. Hence, the coordinate $\rho$ can be used to parametrise the Bondi mass.

Instead of calculating the Bondi mass using the formula (38), we will make use of the fact that

$$
\dot{m}_{B}=-\frac{1}{4 \pi} \oint \dot{\sigma}_{0} \dot{\bar{\sigma}}_{0} d S
$$

and that

$$
\psi_{3}^{0}=i X_{+} \dot{\bar{\sigma}}_{0},
$$

where $\widetilde{\psi}_{3}=\psi_{3}^{0} \Theta^{2}+\cdots$ - see e.g. [24]. The coefficient $\psi_{3}^{0}$ has spin weight 1 , and thus it follows that in this case the operator $X_{+}$has a uniquely defined inverse $X_{+}^{-1}-$ see e.g. [10. Now, from the discussion given in [19] to calculate the Newman-Penrose constants in the F gauge, it also follows that

$$
\widetilde{\psi}_{3}^{0}=\frac{1}{\theta} \Lambda^{a}{ }_{0} \Lambda^{b}{ }_{1} \Lambda^{c}{ }_{1} \Lambda^{d}{ }_{1} \phi_{a b c d} .
$$

Now, only terms with $T_{0}{ }_{0}^{0}$ survive this integration of equation (39). A final integration along the generators of $\mathscr{I}^{+}$yields the following result.

Theorem 5 (Expansions for the Bondi mass). Under the assumptions (i)-(iii) one has that

$$
m_{B}=m-K \sum_{k=0}^{4}\left|\Upsilon_{k}^{(5)}\right|^{2} \rho^{7}+\mathcal{O}\left(\rho^{8}\right),
$$

where $K$ is a numeric factor. If $\Upsilon_{k}^{(5)}=0, k=0, \ldots, 4$ then

$$
m_{B}=m-K^{\prime} \sum_{k=0}^{6}\left|\Upsilon_{k}^{(6)}\right|^{2} \rho^{9}+\mathcal{O}\left(\rho^{10}\right)
$$

where $K^{\prime}$ is a numeric factor. If $\Upsilon_{k}^{(6)}=0, k=0, \ldots, 6$ then

$$
m_{B}=m+\mathcal{O}\left(\rho^{11}\right)
$$

These results are the natural extension of those presented in [26].

\section{Conclusions}

The results presented in this article constitute what we believe is fair evidence to conjecture that the only time reflexion symmetric spacetimes -i.e. those arising as the developments of time symmetric initial data sets - with a smooth conformal extension to null infinity are those that are static in a neighbourhood of spatial infinity.

We would like to contrast the situation of the gravitational field with that of the spin 2 zero rest mass field on a Minkowski background. As shown in [18, 25], an analogue of the regularity condition (11) is a necessary and sufficient condition to obtain logarithm-free solutions to the transport equations, therefore ensuring that the field is smooth at null infinity - the analogue of Penrose's proposal for linear fields. It would be interesting to analyse what happens in the case of fields propagating on curved backgrounds.

The physical intuition suggests that staticity near spatial infinity is also a statement about the behaviour of the gravitational field - and their sources - at very early and late times - that is, near $i^{ \pm}$. This is easier to see in the case of initial data which are Schwarzschildean near spatial infinity. The vanishing of the Newman-Penrose constant implies then in turn the vanishing of the Weyl tensor at $i^{ \pm}$as discussed in the introduction. The interpretation of data which are static near spatial infinity is in any case not that clear for the non-conformally flat case. For as it has been seen, their Newman-Penrose constants do not vanish. Nevertheless, the fact that it is possible to make - non-trivial - statements about the behaviour of spacetimes at $i^{ \pm}$without having to solve the equations is already astonishing. It is worth mentioning that recent results 
by Chruściel \& Delay [2] make possible now to construct initial data sets of the form required by the conjecture presented in this article -i.e. static near $i^{0}$.

Finally, answering the question raised in the title of this article, asymptotic simplicity does seem to exclude gravitational radiation near spatial infinity. A rigorous proof of the conjecture raised here awaits further, deeper, insights into the structure of the (conformal) Einstein field equations.

\section{Acknowledgements}

I would like to thank H. Friedrich who introduced me to this research topic and has provided me with invaluable advice. I also acknowledge several enrichening and helpful discussion with R. Beig, S. Dain and J. Winicour. I also thank an anonymous referee for a careful reading of the manuscript and an important observation leading to lemma 4.

This work is funded by a Lise Meitner fellowship (M690-N02 and M814-N02) of the Fonds zur Forderung der Wissenschaftlichen Forschung (FWF), Austria. The computer algebra calculations here described have been performed in the computer facilities of the Albert Einstein Institute, Max Planck Institute für Gravitationsphysik, Golm, Germany.

\section{References}

[1] A. Ashtekar \& A. Magnon-Ashtekar, Energy-momentum in general relativity, Phys. Rev. Lett. 43, 181 (1979).

[2] P. T. Chruściel \& E. Delay, On mapping properties of the general relativistic constraint operator in weighted function spaces, with applications, in gr-qc/0301073

[3] P. T. Chruściel \& E. Delay, Existence of non-rivial, vacuum, asymptotically simple spacetimes, Class. Quantum Grav. 19, L71 (2002).

[4] P. T. Chruściel, M. A. H. MacCallum, \& D. B. Singleton, Gravitational waves in general relativity XIV. Bondi expansions and the "polyhomogeneity" of $\mathscr{I}$, Phil. Trans. Roy. Soc. Lond. A 350, 113 (1995).

[5] J. Corvino, Scalar curvature deformations and a gluing construction for the Einstein constraint equations, Comm. Math. Phys. 214, 137 (2000).

[6] S. Dain, Private communication.

[7] S. Dain, Initial data for stationary spacetimes near spacelike infinity, Class. Quantum Grav. 18, 4329 (2001).

[8] S. Dain \& H. Friedrich, Asymptotically flat initial data with prescribed regularity at infinity, Comm. Math. Phys. 222, 569 (2001).

[9] S. Dain \& J. A. Valiente Kroon, Conserved quantities in a black hole collision., Class. Quantum Grav. 19, 811 (2002).

[10] A. R. Exton, E. T. Newman, \& R. Penrose, Conserved quantites in the Einstein-Maxwell theory, J. Math. Phys. 10, 1566 (1969).

[11] H. Friedrich, Radiative gravitational fields and asymptotically static or stationary initial data, to appear in the volume "50 years of the Cauchy problem in general relativity", Birkhauser. in $\mathrm{gr}-\mathrm{qc} / 0304003$

[12] H. Friedrich, On the existence of n-geodesically complete or future complete solutions of Einstein's field equations with smooth asymptotic structure, Comm. Math. Phys. 107, 587 (1986).

[13] H. Friedrich, On static and radiative space-times, Comm. Math. Phys. 119, 51 (1988). 
[14] H. Friedrich, Einstein equations and conformal structure: existence of anti-de Sitter-type space-times, J. Geom. Phys. 17, 125 (1995).

[15] H. Friedrich, Gravitational fields near space-like and null infinity, J. Geom. Phys. 24, 83 (1998).

[16] H. Friedrich, Einstein's equation and conformal structure, in The Geometric Universe. Science, Geometry and the work of Roger Penrose, edited by S. A. Huggett, L. J. Mason, K. P. Tod, S. T. Tsou, \& N. M. J. Woodhouse, page 81, Oxford University Press, 1999.

[17] H. Friedrich, Conformal Einstein evolution, in The conformal structure of spacetime: Geometry, Analysis, Numerics, edited by J. Frauendiener \& H. Friedrich, Lecture Notes in Physics, page 1, Springer, 2002.

[18] H. Friedrich, Spin-2 fields on Minkowski space near space-like and null infinity, Class. Quantum Grav. 20, 101 (2003).

[19] H. Friedrich \& J. Kánnár, Bondi-type systems near space-like infinity and the calculation of the NP-constants, J. Math. Phys. 41, 2195 (2000).

[20] H. Friedrich \& B. Schmidt, Conformal geodesics in general relativity, Proc. Roy. Soc. Lond. A 414, 171 (1987).

[21] E. T. Newman \& R. Penrose, New conservation laws for zero rest-mass fields in asymptotically flat space-time, Proc. Roy. Soc. Lond. A 305, 175 (1968).

[22] R. Penrose, Zero rest-mass fields including gravitation: asymptotic behaviour, Proc. Roy. Soc. Lond. A 284, 159 (1965).

[23] P. Sommers, Space spinors, J. Math. Phys. 21, 2567 (1980).

[24] J. Stewart, Advanced general relativity, Cambridge University Press, 1991.

[25] J. A. Valiente Kroon, Polyhomogeneous expansions close to null and spatial infinity, in The Conformal Structure of Spacetimes: Geometry, Numerics, Analysis, edited by J. Frauendiner \& H. Friedrich, Lecture Notes in Physics, page 135, Springer, 2002.

[26] J. A. Valiente Kroon, Early radiative properties of the developments of time symmetric conformally flat initial data, Class. Quantum Grav. 20, L53 (2003).

[27] J. A. Valiente Kroon, A new class of obstructions to the smoothness of null infinity, Comm. Math. Phys. 244, 133 (2004).

[28] J. A. Valiente Kroon, On the nonexistence of conformally flat slices for the Kerr and other stationary spacetimes, Phys. Rev. Lett. 92, 041101 (2004). 